\title{
Erratum
}

\section{Erratum to : Visualizing Electromagnetic Vector Fields in Matter Using MATHEMATICA}

\section{Received December 28, 2018; accepted February 27, 2019}

\section{Yong-Dae Choi and Hee-Joong Yun*}

Department of Microbial \& Nanomaterials, Mokwon University, Daejeon 35349, Republic of Korea

College of Techno-Sciences, Mokwon University, Daejeon 35349, Republic of Korea

*Corresponding author E-mail: heejy@mokwon.ac.kr

This erratum is being published to correct error on pages [pp.67-75] of the article [vol 28, No 3] entitled "Visualizing Electromagnetic Vector Fields in Matter using Mathematica".

p.67 right last line: $\vec{p}$ should be corrected to $\vec{P}$

p.68 left 18th line: "having" should be corrected to "have"

p.68 right 9th line : $\left|r_{2}-r_{1}\right|^{-1}=\frac{1}{r_{2}}\left[1+\frac{r_{1} \cdot r_{2}}{r_{2}^{2}}+\ldots\right]$ corrected to $\left|r_{2}-r_{1}\right|^{-1}=\frac{1}{r_{2}}\left[1+\frac{\vec{r}_{1} \cdot \vec{r}_{2}}{r_{2}^{2}}+\ldots\right]$

p.70 left 9th line: $2 I 0_{x} ; 2 I 0_{y}$ to $2 I_{0} x ; 2 I_{0} y$

p.71 left 3rd line: $A_{1} a^{1}=B_{1} \frac{1}{a^{1+1}}$ to $A_{l} a^{l}=B_{l} \frac{1}{a^{l+1}}$ and 4th line: $A_{1}=\frac{P}{3_{\epsilon 0}}$ to $A_{1}=\frac{P}{3 \epsilon_{0}}$

p.71 right, 4th line: huefunc $=\frac{\text { Norm }[v f u n c]}{v f \max }$ to huefunc $=\frac{\text { Norm }[v f u n c]}{v f \max }$

p.72 left 5th line: $r \geq a$ to $r \leq a$

p.72 right 2nd line: $r \geq a$ to $r \leq a$

p.73 left, Eq.(29) : $\phi_{M}(r, \theta)=\frac{1}{3} M a^{2} \frac{r<}{r_{2}>} \operatorname{Cos}[\theta]$ to $\phi_{M}(r, \theta)=\frac{1}{3} M a^{2} \frac{r_{<}}{r_{>}^{2}} \operatorname{Cos}[\theta]$

p.73 left, Eq.(31) $: r \geq a$ to $r \leq a$

p.74 right, 1st line : $\operatorname{In}[451-458]$ to $\operatorname{In}[451-458]$ in MatheMatica code1

p.75 right, 31st line : "intensity" to "intensity array"

p.75 right, 4th line : $\vec{E}_{2}=-\frac{P}{3} \hat{e} z$ should be corrected to $\vec{E}_{2}=-\frac{P}{3} \hat{e}_{z}$ 